\title{
Optical observation of single-carrier charging in type-Il quantum ring ensembles
}

\author{
R. J. Young, ${ }^{1, a)}$ E. P. Smakman, ${ }^{2}$ A. M. Sanchez, ${ }^{3}$ P. Hodgson, ${ }^{1}$ P. M. Koenraad, ${ }^{2}$ \\ and M. Hayne ${ }^{1}$ \\ ${ }^{1}$ Department of Physics, Lancaster University, Lancaster LA1 4YB, United Kingdom \\ ${ }^{2}$ Department of Applied Physics, Eindhoven University of Technology, The Netherlands \\ ${ }^{3}$ Department of Physics, Warwick University, Coventry CV4 7AL, United Kingdom
}

(Received 3 January 2012; accepted 2 February 2012; published online 22 February 2012)

\begin{abstract}
A high-purity $\mathrm{GaSb} / \mathrm{GaAs}$ quantum ring system is introduced that provides both strong hole-confinement in the GaSb ring and electron confinement in its GaAs core. The latter is responsible for a reduced inhomogeous linewidth measured in photoluminescence, in comparison to the previous measurements made on nanostructures with differing morphology in this material system. This allows the resolution of multiple peaks in the photoluminescence due to discrete charging with holes, revealing the mechanism responsible for the excitation-power-induced blueshift. (C) 2012 American Institute of Physics. [doi:10.1063/1.3688037]
\end{abstract}

Semiconductor nanostructures ${ }^{1}$ are appealing for applications in quantum information processing (QIP) (Ref. 2), as they provide an interface between flying- and stationaryqubits. ${ }^{3}$ Two significant physical limitations are present in most systems: shallow confinement requires low-temperature operation and strong interactions with their surroundings typically result in short coherence times. ${ }^{4,5} \mathrm{GaSb} / \mathrm{GaAs}$ structures ${ }^{6}$ could potentially address these limitations, as they present a deep confining potential for holes ${ }^{7}$ making room-temperature operation possible, ${ }^{8}$ and hole-spins can be robust against dephasing. ${ }^{9,10}$ The type-II nature of this system is non-ideal, however, and has led to inferior optical properties. ${ }^{11}$ The excitonic physics of type-II quantum dot systems, in which just one of the two carrier types is confined, is known to be strikingly different from that of type-I systems where both are. ${ }^{12}$ For a neutral multi-exciton state in a type-II nanostructure, the strongly confined carrier type will experience a much greater Coulomb repulsion than would be expected in a type-I system, due to the close proximity of like-, and absence of oppositely, charged carriers. In this letter, the significance of the Coulomb interaction in a type-II system is directly demonstrated by optical measurement of the discrete charging of ensembles of $\mathrm{GaSb} / \mathrm{GaAs}$ quantum rings with holes.

Molecular-beam-epitaxial (MBE) growth was used to form dislocation-free rings with a very high material contrast, as shown in Fig. 1. The experimental procedure used to control the morphology of $\mathrm{GaSb} / \mathrm{GaAs}$ nanostructures formed during growth by molecular beam epitaxy was introduced in Ref. 13. MBE growth tends to result in the formation of rings, rather than dots, ${ }^{14}$ with the final capping, or annealing, temperature influencing the ratio of major/minor axes of the rings that form. ${ }^{15}$ The primary sample grown for this study comprised of a single layer of GaSb quantum rings capped with $250 \mathrm{~nm}$ of GaAs. The ring layer was grown by depositing 2.1 monolayers (MLs) of GaSb at a pyrometermeasured temperature of $490^{\circ} \mathrm{C}$ with a growth rate of 0.3 $\mathrm{MLs}^{-1}$ and a V/III ratio of approximately 10 . This layer was capped with a $5 \mathrm{~nm}$ layer of GaAs at $430^{\circ} \mathrm{C}$ prior to growing

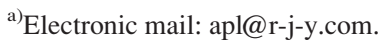

the remaining $245 \mathrm{~nm}$ of cap at $1 \mathrm{MLs}^{-1}$ at a temperature of $580^{\circ} \mathrm{C}$. The band-structure of $\mathrm{GaSb} / \mathrm{GaAs}$ quantum rings is illustrated in Ref. 6. Samples containing layers of rings were also grown under similar conditions for cross-sectional microscopy; both high-resolution transmission electron microscopy (HRTEM) and scanning tunneling microscopy (STM) were employed to assess the structural result of growth (Fig. 1). As the latter technique requires a tunnel current to facilitate local density of state measurements, a low level of background doping (n-type $\sim 10^{17} \mathrm{~cm}^{-3}$ using GaTe) was introduced to the growth of all layers in the sample. The image in Fig. 1(d) was taken with a tip bias of $+0.44 \mathrm{~V}$.

When the rings are measured in cross-section two GaSbrich lobes are typically seen, with separations varying between 0 and $\sim 25 \mathrm{~nm}$, the value measured is dependent on the position of the section with respect to the center of the ring (Fig. 1(c)). The major radius of the rings is unlikely to influence the heavy-hole's wave-function in this system, as it is expected to be localized in a relatively small section of the ring. Despite this, the geometry does strongly influence the excitonic properties in the system. STM measurements indicate that the $\mathrm{GaAs}$ in the center of the ring is purer, i.e., contains less $\mathrm{Sb}$, than that surrounding it. A local electron density-of-states measurement confirms the presence of a maximum towards the center of the ring, as shown in Fig. 1(d).

Optical measurements on the quantum rings were performed at $1.4 \mathrm{~K}$ by placing the nominally undoped sample in a ${ }^{4} \mathrm{He}$ cryostat. Stabilized $532 \mathrm{~nm}$ continuous-wave laser light was delivered to the sample, via an optical fiber with a large-diameter core, to excite carriers above the GaAs bandgap. Photoluminescence (PL) was collected from an area corresponding to $\sim 2 \mathrm{~mm}^{2}$ using a second optical fiber. Microscopy estimated the ring areal number density to be of the order of $1 \times 10^{10} \mathrm{~cm}^{-2}$. The sample's emission was passed into a $0.3 \mathrm{~m}$ spectrometer equipped with a Peltier-cooled InGaAs array with which multi-channel wavelength-resolved measurements were made. PL from the rings taken at low (high) excitation power is shown in (the inset of) Fig. 2. Emission from the nanostructures is strong when compared 

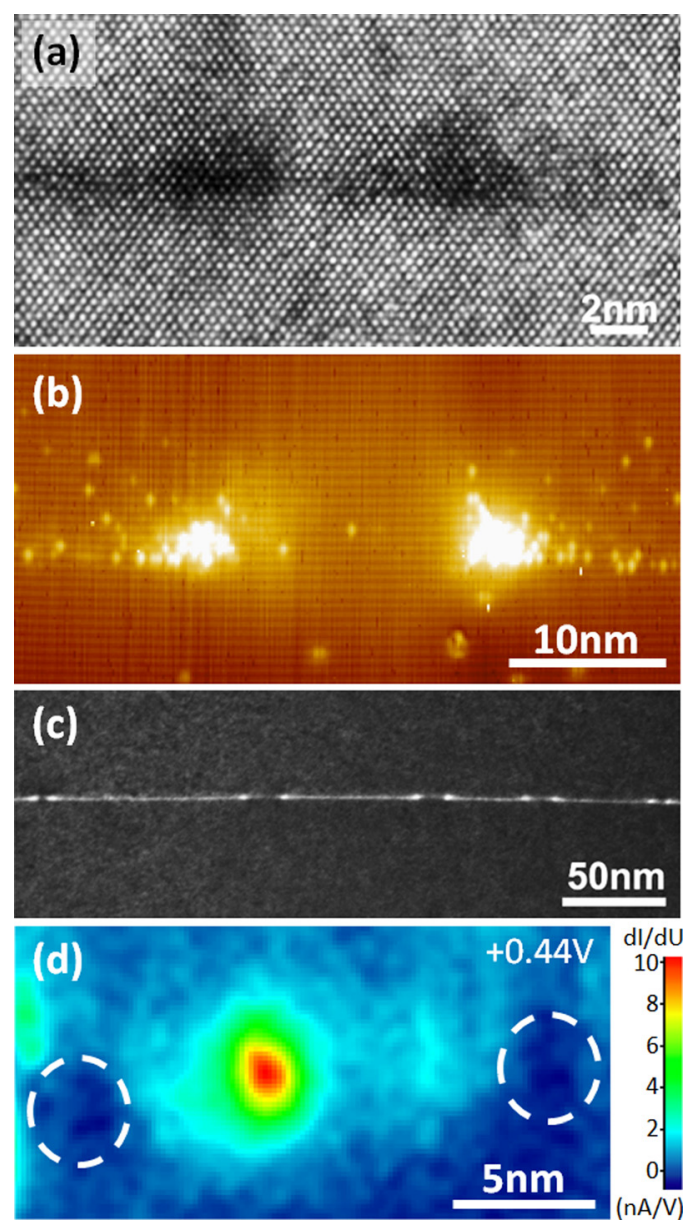

FIG. 1. (Color online) (a) High-resolution transmission electron microscope (TEM) and (b) filled-state scanning tunneling microscopy images, illustrating excellent material contrast between GaAs and GaSb rich regions and showing no crystal defects. (c) Low magnification dark field 002 TEM showing several GaSb various quantum rings (light) in the GaAs (dark) matrix. (d) An electronic density-of-states image of a similar ring taken using a STM reveals a maximum towards the center of the ring. The dashed ellipses mark GaSb-rich lobes, as a guide. All images were taken in cross-section.

to other emitters in the sample, indicating that the rings have good optical properties. The peak emission energy of the rings is below $1 \mathrm{eV}$ demonstrating the very strong confining potential for this material system. ${ }^{7}$ The peak also contains clear oscillatory features that were investigated further by monitoring their evolution as a function of the excitation power, as shown in Fig. 3.

The center of mass $(\mathrm{CoM})$ of the emission from the rings blueshifts with increasing pump intensity by more than $50 \mathrm{meV}$ over the five orders of magnitude of power probed here. An excitation-power-induced blueshift is a characteristic feature that has been reported in numerous different quantum-confining type-II systems. A bending of the bandstructure $^{16}$ at the interface between the different carrier types and increased charging by the strongly confined carrier are both thought to contribute to this shift. In quantum dots, however, the latter is thought to play a much greater role than the former. ${ }^{17}$ The oscillations in the PL were clearly visible at all powers probed.

The quantum ring PL data was analyzed by fitting a series of fixed-width Gaussian sub-peaks to each of the measured spectra, an example of this fitting is illustrated in the inset of Fig. 2. This fit closely matched the experimental data through-

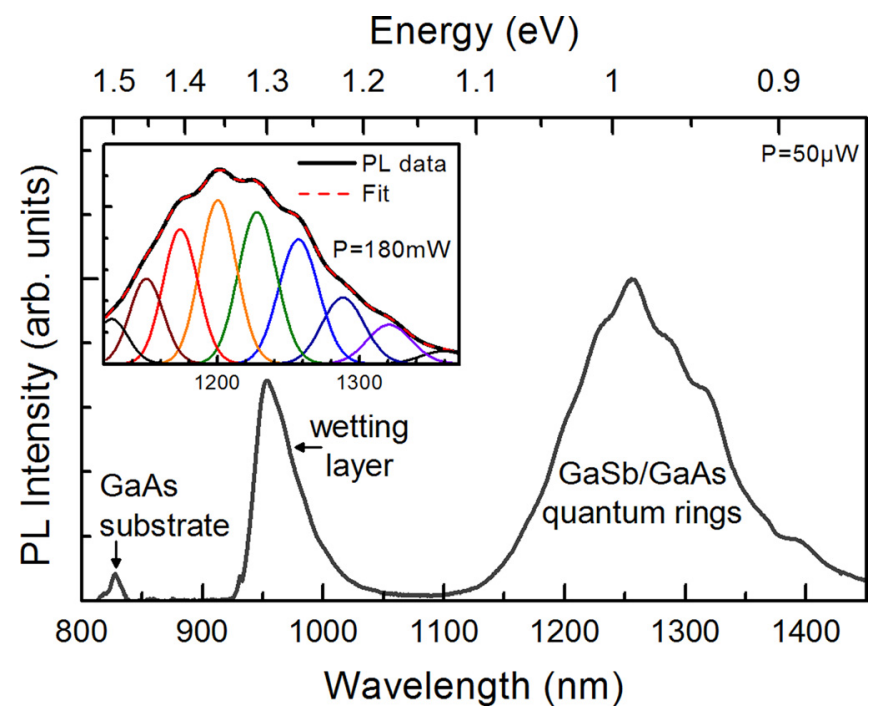

FIG. 2. (Color online) Emission from the rings dominates the photoluminescence spectrum; clear oscillations due to discrete charging can be seen. The inset shows the ring-peak under high-excitation power alongside a fit to the data using multiple Gaussian sub-peaks.

out the power-range studied. The sub-peak centers and areas are plotted as a function of power in Figs. 3 (lower panel) and 4 , respectively. The width of the Gaussians used in the fitting procedure was $26 \mathrm{meV}$, and the separation between the subpeaks was found to increase from $19 \mathrm{meV}$ between the lowest energy sub-peaks to $23 \mathrm{meV}$ between the high energy subpeaks. The former, i.e., $26 \mathrm{meV}$, is believed to be an upper limit to the inhomogeneous broadening in the system, while the latter represents the energy required to overcome the repulsive Coulomb interaction when adding a further heavyhole to the dot; justification for this statement follows.

A simulation ${ }^{17}$ of $\mathrm{Ga}(\mathrm{As}) \mathrm{Sb} / \mathrm{GaAs}$ quantum dots included the Coulomb potential in an 8-band $\boldsymbol{k} . \boldsymbol{p}$ model. As the dot was filled with holes, the ground-state (E1-H1)

(a)
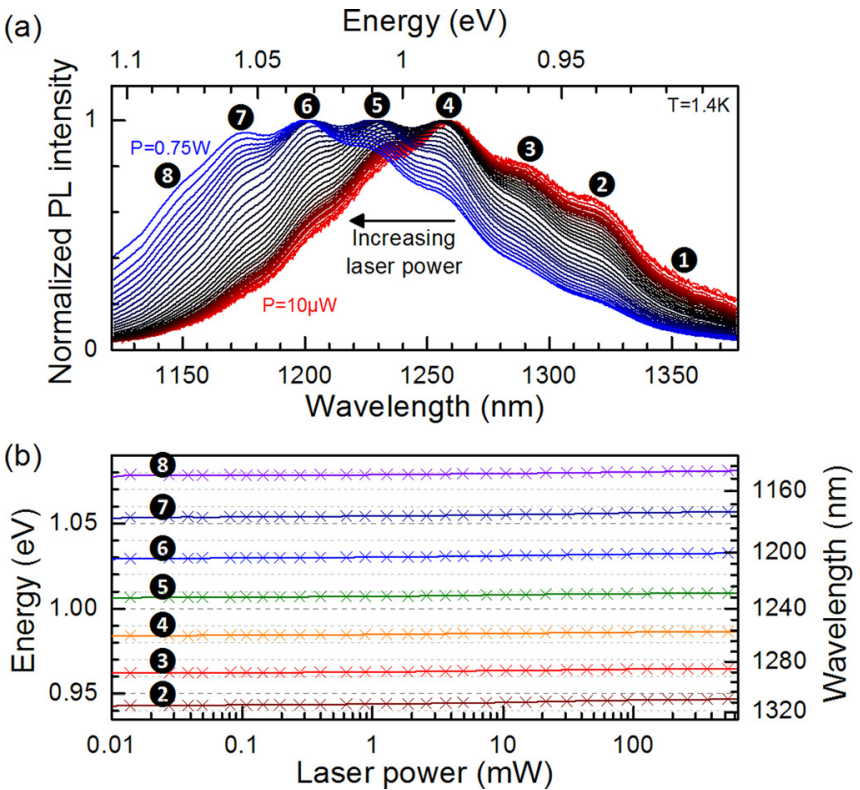

FIG. 3. (Color online) (a) The peak in photoluminescence associated with emission from the quantum rings blue-shifts strongly as the laser power is increased by five orders of magnitude. Sub-peaks are labeled with the discrete hole populations they represent. (b) The energetic evolution of individual hole populations; a weak blue-shift is visible. 


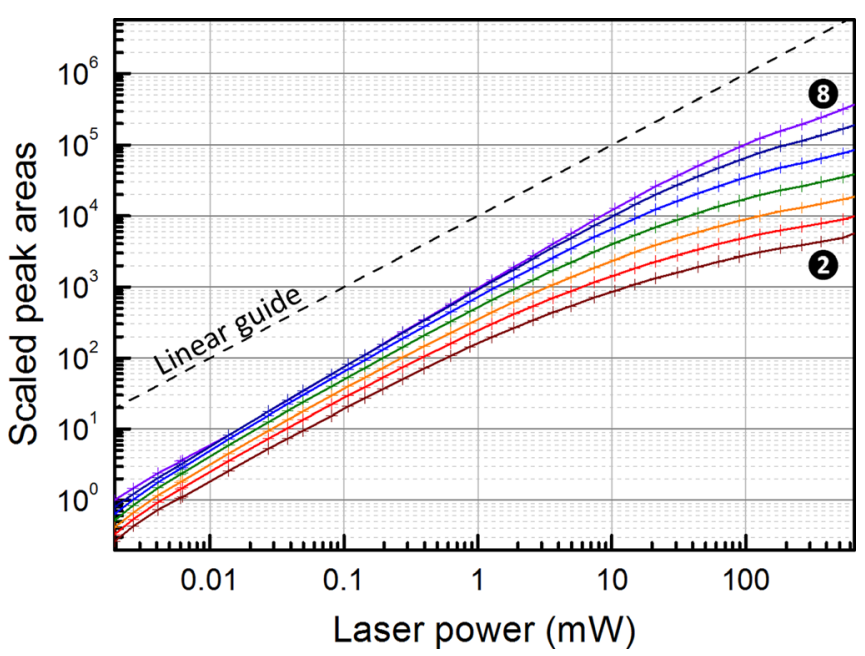

FIG. 4. (Color online) Emission from states containing fewer holes saturates earlier, showing sub-linear power dependencies at lower powers and resulting in a strong blue-shift. The curves have been scaled and displaced evenly on the log-scale at lowest power to allow comparison of their power dependencies.

transition was found to blue-shift strongly as a result of the Coulomb interaction. The interpretation of our experimental data is somewhat similar, though the magnitude of the effect is several times greater. Reference 17 studied rather large $(25 \times 25 \times 15 \mathrm{~nm})$ dilute $(25 \% \mathrm{Sb})$ pyramidal dots, whereas, in this work, the hole-confining region is much smaller (see Fig. 1) and the Sb concentration in the ring is close to $100 \%$. One possible alternative explanation for the sub-peaks is recombination from the excited states of the rings. However, if this explanation were true, a highly non-linear behavior of the peak intensity with power would be expected as higher energy states become rapidly occupied at increasing excitation power; this is not observed (see Fig. 4). Recombination from excited hole states ${ }^{11}$ and light-hole states are likely to be present in the measured PL, but not resolved. A second possible explanation for the sub-peaks is monolayer fluctuations ${ }^{18}$ in the size of the rings. If this were the case, the lower energy emission from larger structures would be expected to saturate at higher powers than the higher energy emission from smaller structures. Again, this is not seen in our data where the majority of the blueshift of the CoM is a result of the lower energy states saturating at smaller laser powers than the higher energy states. The sub-peaks could also potentially be explained by Fabry-Pérot modes, but this explanation is not consistent with the power-induced shift of these peaks, and substructure is not observed in the emission from the wetting layer. We conclude that the substructure in the PL from the quantum rings is most likely due to discrete hole charging.

Concerning band-bending, ${ }^{16}$ emission from exciton states containing a fixed number of holes, i.e., the sub-peaks in the PL spectrum, is found to blue-shift with a magnitude that is much less than the CoMs shift (Fig. 3(b)). This is attributed to a collective band-bending effect as a result of an increase in the number of electrons that surround the rings and charging of the wetting layer.

Recent 8-band $\boldsymbol{k} . \boldsymbol{p}$ calculations have shown that the maximum localization energy of a pure GaSb/GaAs nanostructure with similar dimensions to those studied here is approximately $600-650 \mathrm{meV} \cdot{ }^{15,19}$ With a low-temperature GaAs band-gap of $\sim 1.5 \mathrm{eV}$, emission from the excitonic state in which just one heavy-hole occupies the dot should not occur below $0.9 \mathrm{eV}$. This allows the number of holes in each of the PL peaks to be quantified, as indicated by the numbers in circles in Figs. 3 and 4, and allows important observations to be made about this ring system. The peak corresponding to an occupancy of one hole/ring is difficult to fit due to its proximity to an absorption-dip in the optical fiber which is used to collect the emission from the sample. As the pump power tends towards zero, emission appears preferentially from the excitonic states with 4 , rather than 1 , holes/ring. This indicates that a degree of unintentional doping is present in the sample. A dependence of the exciton's oscillator strength on the number of holes in a ring may contribute to this, though unintentional incorporation of carbon acceptors, as indicated by the presence of an acceptor-bound GaAs peak in the PL measurements, is thought to be the primary cause. Such background doping has been measured previously in the growth of GaSb nanostructures. ${ }^{20}$

The GaSb/GaAs quantum rings introduced in this work have unique physical properties, linked directly to their material properties, which may make them useful for applications in QIP. Unlike most zero-dimensional type-II systems, here the "unbound" carrier type does experience a confining potential that is spatially close to that of the bound carriers. This is believed to be responsible for the reduction in emission line-width of the ensemble that facilitated the measurement of discrete charging. It is also expected to lead to an increase in the exciton's optical oscillator strength, though tools required to measure this were not available in this study. Weak oscillator strengths and broad line-widths have disincentivised research on individual type-II nanostructures; this system may help to address these issues. GaSb/GaAs has two significant advantages over widely researched InAs/ GaAs dots; it has a small nuclear moment and sufficient localization energy to provide confinement well above room temperature. ${ }^{8}$ It may have a promising future for applications manipulating and storing individual hole-spins.

This work was supported by the FOM and the Engineering and Physical Sciences Research Council (Grant No. EP/ H006419), in the framework of the QD2D project, the Royal Society-Brian Mercer Feasibility Award, and by QinetiQ (agreement 3000127730). A.M.S. would like to acknowledge the Science City Research Alliance and the HEFCE Strategic Development Fund for funding support.

${ }^{1}$ F. Henneberger and O. Benson, Semiconductor Quantum Bits (Pan Stanford, Singapore, 2009).

${ }^{2}$ A. J. Shields, Nature Photon. 1, 215 (2007).

${ }^{3}$ A. Boyer de la Giroday, N. Sköld, R. M. Stevenson, I. Farrer, D. A. Ritchie, and A. J. Shields, Phys. Rev. Lett. 106, 216802 (2006).

${ }^{4}$ A. V. Khaetskii, D. Loss, and L. Glazman, Phys. Rev. Lett. 88, 186802 (2008).

${ }^{5}$ J. R. Petta, A. C. Johnson, J. M. Taylor, E. A. Laird, A. Yacoby, M. D. Lukin, C. M. Marcus, M. P. Hanson, and A. C. Gossard, Science 309, 2180 (2009).

${ }^{6}$ R. Timm, H. Eisele, A. Lenz, L. Ivanova, V. Vossebürger, T. Warming, D. Bimber, I. Farrer, D. A. Ritchie, and M. Dähne, Nano Lett. 10, 3972 (2010). ${ }^{7}$ M. Hayne, J. Maes, S. Bersier, V. V. Moshchalkov, A. Schliwa, L. MüllerKirsch, C. Kapteyn, R. Heitz, and D. Bimberg, Appl. Phys. Lett. 82, 4355 (2003). 
${ }^{8}$ F. Hatami, N. N. Ledentsov, M. Grundmann, J. Böhrer, F. Heinrichsdorff, M. Beer, D. Bimberg, S. S. Ruvimov, P. Werner, U. Gösele et al., Appl. Phys. Lett. 67, 656 (1995).

${ }^{9}$ B. D. Gerardot, D. Brunner, P. A. Dalgarno, P. Öhberg, S. Seidl, M. Kroner, K. Karrai, N. G. Stoltz, P. M. Petroff, and R. J. Warburton, Nature 451, 441 (2008).

${ }^{10}$ D. Brunner, B. D. Gerardot, P. A. Dalgarno, G. Wüst, K. Karrai, N. G. Stoltz, P. M. Petroff, and R. J. Warburton, Science 325, 70 (2009).

${ }^{11}$ K. Gradkowski, N. Pavarelli, T. J. Ochalski, D. P. Williams, J. Tatebayashi, G. Huyet, E. P. O'Reilly, and D. L. Huffaker, Appl. Phys. Lett. 95, 061102 (2009).

${ }^{12}$ B. Bansal, M. Hayne, M. Geller, D. Bimberg, and V. V. Moshchalkov, Phys. Rev. B 77, 241304 (2004).

${ }^{13}$ M. Ahmad Kamarudin, M. Hayne, R. J. Young, Q. D. Zhuang, T. Ben, and S. I. Molina, Phys. Rev. B 83, 115311 (2011).

${ }^{14}$ R. Timm, H. Eisele, A. Lenz, L. Ivanova, G. Balakrishnan, D. L. Huffaker, and M. Dähne, Phys. Rev. Lett. 101, 256101 (2008).
${ }^{15}$ E. P. Smakman, J. K. Garleff, R. J. Young, M. Hayne, P. Rambabu, and P. M. Koenraad, "GaSb/GaAs Quantum Dot Formation and Demolition Studied with Cross-Sectional Scanning Tunneling Microscopy" (unpublished).

${ }^{16}$ N. N. Ledentsov, J. Böhrer, M. Beer, F. Heinrichsdorff, M. Grundmann, D. Bimberg, S. V. Ivanov, B. Ya Meltser, S. V. Shaposhnikov, I. N. Yassievich et al., Phys. Rev. B 52, 14058 (1995).

${ }^{17}$ K. Gradkowski, T. J. Ochalski, D. P. Williams, S. B. Healy, J. Tatebayashi, G. Balakrishnan, E. P. O'Reilly, G. Huyet, and D. L. Huffaker, Phys. Status Solidi B 246, 752 (2009).

${ }^{18}$ J. Maes, M. Hayne, Y. Sidor, B. Partoens, F. M. Peeters, Y. González, L. González, D. Fuster, J. M. Garcia, and V. V. Moshchalkov, Phys. Rev. B 70, 155311 (2004).

${ }^{19}$ T. Nowozin, A. Marent, L. Bonato, A. Schliwa, D. Bimberg, E. P. Smakman, J. Garleff, R. Pachineela, P. M. Koenraad, R. J. Young et al., "Structural, electrical and theoretical investigations of high-purity selfassembled $\mathrm{GaSb} / \mathrm{GaAs}$ quantum dots" (unpublished).

${ }^{20}$ M. Hayne, O. Razinkova, S. Bersier, R. Heitz, L. Müller-Kirsch, M. Geller, D. Bimberg, and V. V. Moshchalkov, Phys. Rev. B. 70, 081302 (2004). 